\title{
Band Engineering and Magnetic Doping of Epitaxial Graphene on SiC (0001)
}

\author{
Thushari Jayasekera, ${ }^{1}$ B. D. Kong, ${ }^{2}$ K. W. Kim, ${ }^{2}$ and M. Buongiorno Nardelli ${ }^{1,3, *}$ \\ ${ }^{1}$ Department of Physics, North Carolina State University, Raleigh, North Carolina 27695-7518, USA \\ ${ }^{2}$ Department of Electrical and Computer Engineering, North Carolina State University, Raleigh, North Carolina 27695-7911, USA \\ ${ }^{3}$ Computer Science and Mathematics Division, Oak Ridge National Laboratory, Oak Ridge, Tennessee 37831-6359, USA
}

(Received 13 December 2009; published 5 April 2010)

\begin{abstract}
Using calculations from first principles we show how specific interface modifications can lead to a finetuning of the doping and band alignment in epitaxial graphene on SiC. Upon different choices of dopants, we demonstrate that one can achieve a variation of the valence band offset between the graphene Dirac point and the valence band edge of $\mathrm{SiC}$ up to $1.5 \mathrm{eV}$. Finally, via appropriate magnetic doping one can induce a half-metallic behavior in the first graphene monolayer. These results clearly establish the potential for graphene utilization in innovative electronic and spintronic devices.
\end{abstract}

DOI: 10.1103/PhysRevLett.104.146801

Advances in the epitaxial growth of graphene films on $\mathrm{SiC}$ have the potential to open new classes of device applications that may revolutionize the semiconductor road map for future decades [1-9]. However, this progress will require an in-depth understanding and utilization of the electronic processes that take place at the nanoscale, in particular, the role of the interface buffer layer, where most of the electronic properties of the system can be controlled [10]. In analogy with the formation of the Schottky barrier in metal-insulator interfaces here we demonstrate the ability to tune and control the band alignment and the magnetic doping at the heterojunction between graphene and $\mathrm{SiC}$ $[11,12]$. Epitaxial growth of graphene on $\mathrm{SiC}$ is obtained by vacuum graphitization due to $\mathrm{Si}$ depletion of the $\mathrm{SiC}$ single crystal surface [13]. This method has been shown to produce ultrathin carbon sheets and occasionally a graphene monolayer or bilayer [14-17]. Most importantly, the first interfacial layer of carbon grown on $\mathrm{SiC}$ differs substantially from the ideal graphene structure. Its geometry is dictated by the surface atomic configuration and it acts as a buffer layer between the substrate and the layers above [2]. Moreover, the very existence of this buffer layer suggests the possibility of using it for manipulation of electronic properties of the heterojunction. This interface phase can act as a "Coulomb buffer" [11], where the structure specifics of heteroepitaxy can be used to set the electrostatic and magnetic boundary conditions of the overall system.

In this Letter we will show, using calculations from first principles, how the surface of the substrate and the buffer layer can be modified to allow for the fine-tuning of the doping and of the band alignment between the graphene Dirac point and the valence band edge of $\mathrm{SiC}$ and, more importantly, how, via specific magnetic doping, one can induce a half-metallic behavior in the first graphene monolayer that demonstrate the potential for graphene utilization in innovative spintronic devices.

Graphene is nearly commensurate with the $6 \sqrt{3} \times$ $6 \sqrt{3} \cos \left(30^{\circ}\right)(6 R 3)$ structure of $\mathrm{SiC}(0001)$ surface [7].
PACS numbers: 73.22.Pr, 61.48.Gh, 71.15.Mb, 81.05.ue

In this Letter, we consider the $\sqrt{3} \times \sqrt{3} \cos 30^{\circ}(R 3)$ structure [18]. The unit cell of the $R 3$ structure contains $6 \mathrm{SiC}$ bilayers as in $6 \mathrm{H}$-SiC. A $2 \times 2$ graphene unit cell is placed on top of this $R 3 \mathrm{SiC}$ cell. In this configuration, the first graphene monolayer above the buffer is stretched by $8 \%$ compared to its equilibrium geometry, thus increasing the elastic energy of the system. However, this energy is reduced by the subsequent relaxation of the graphene layer on the substrate [7]. Moreover, we expect that the buffer layer, being strongly bonded with the $\mathrm{SiC}$ substrate will be accurately described by this choice of geometry.

All calculations were performed in the framework of density functional theory (DFT) as it is implemented in the QUANTUM-ESPRESSO package [19]. The optimized geometry of $2 \times 2$ unit cells and the structure of the buffer layer are shown in Fig. 1. There are $3 \mathrm{Si}$ atoms on the surface of the $R 3$ unit cell that can be classified according to their chemical environment. Two of them are chemically bonded to the $\mathrm{C}$ atoms in the first $\mathrm{C}$ layer, while the third one lies exactly underneath the center of the $\mathrm{C}$ hexagons on the top. In this idealized configuration, the third $\mathrm{Si}$ atom does not form any bonding with the layer above and it is characterized by a single unbound electron. Following the current convention, we will refer to the former as the $\mathrm{Si}_{b}$ (bonded) and the latter as $\mathrm{Si}_{l}$ (lonely). Because of the covalent bonding between the $\mathrm{Si}_{b}$ and the $\mathrm{C}$ atoms, a delocalized $\pi$-orbital structure is not possible for the buffer layer on the substrate. This $\mathrm{C}$ layer will in fact not show any of the electronic properties of ideal graphene [18]. The next layers on top of the buffer layer are bound by weak van der Waals forces and in turn will show graphenic electronic properties. We will denote the buffer layer atoms as $\mathrm{C}_{b}$ and the first graphene layer atoms as $\mathrm{C}_{g}$.

The vertical distance between the $\mathrm{Si}_{b}$ and the $\mathrm{C}_{b}$ layer is $2.0 \AA$ and the distance between the $\mathrm{Si}_{l}$ atom and the closest $\mathrm{C}$ atom in the top $\mathrm{C}_{b}$ layer is $2.65 \AA$. The distance between $\mathrm{C}_{b}$ layer and the $\mathrm{C}_{g}$ layer varies between $3.90-4.2 \AA$. The variation of the distance between the layers at different sites is due to the nonplanar rippled structure of the buffer 


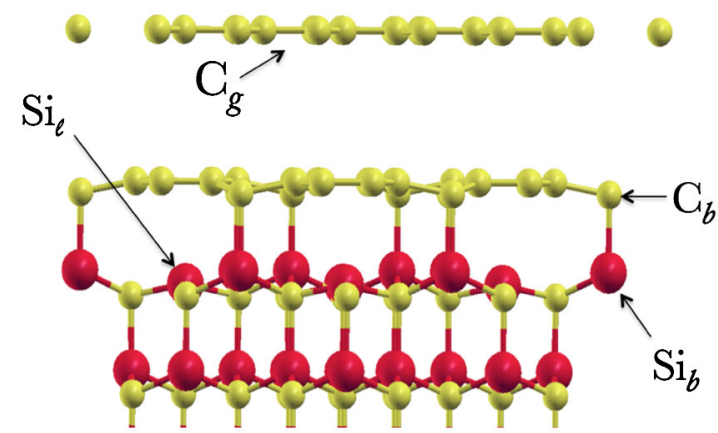

FIG. 1 (color online). Side view of the optimized geometry of $2 \times 2$ hexagonal unit cell of the graphene/C buffer layer/SiC (0001) system. Red (dark) color atoms are $\mathrm{Si}$ and the yellow (light) color are $\mathrm{C}$ atoms. The first graphene layer $\left(c_{g}\right)$, buffer layer $\left(c_{b}\right)$ and the last two $\mathrm{SiC}$ bilayers of the substrate are shown. The two types of $\mathrm{Si}$ atoms, $\mathrm{Si}_{l}$ and $\mathrm{Si}_{b}$ are indicated. In the passivated interface geometry, an additional atom is bonded to the $\mathrm{Si}_{l}$ atom in the interstitial region between $\mathrm{SiC}$ and the buffer layer. In the case of substitutional doping, $\mathrm{Si}_{l}$ is replaced by another atomic species.

layer. All the geometrical notations can be found in the Fig. 1. Figure 2(a) shows the electronic band structure for this system: two C layers on top of $\mathrm{SiC}$ (0001). The electronic properties are dominated by the half-filled interface state that is the signature of the dangling electron on the $\mathrm{Si}_{l}$ atom, that maintain the $s p^{3}$ character of its bulk counterpart in the $\mathrm{SiC}$ matrix. This dangling bond is also responsible for the charge transfer of $1.95 \times 10^{13} \mathrm{~cm}^{-2}$ that induces the shift of the Dirac point to $0.4 \mathrm{eV}$ below the Fermi energy $[2,18]$ that effectively pins the Fermi energy.

The presence of the $\mathrm{Si}_{l}$ lonely atoms suggests an obvious route for the functionalization of the interface region and the control of the electrostatics at the junction. Indeed, by manipulating the charge of the $\mathrm{Si}_{l}$ atom, one can modify the microscopic charge distribution (the Coulomb buffer) and tailor the properties of the system. One possibility is in

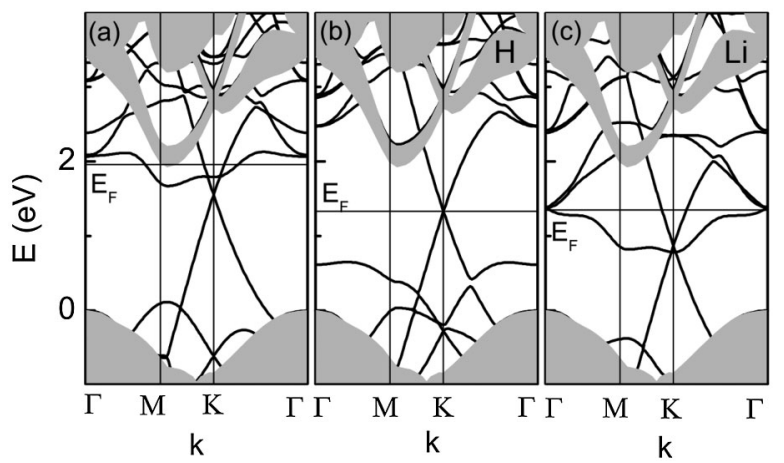

FIG. 2. The electronic band structures of the monolayer graphene and buffer layer on top of $\mathrm{SiC}$ substrate with (a) $\mathrm{Si}_{l}$ is not bonded to any chemical species (intrinsic), (b) $\mathrm{Si}_{l}$ is passivated with a $\mathrm{H}$ atom, and (c) $\mathrm{Si}_{l}$ atom is passivated with $\mathrm{Li}$ atom. The shaded area is the surface projected bulk band structure of $\mathrm{SiC}$ and all the energies are measured relative to the maximum of the valence band in bulk $\mathrm{SiC}$. exploiting the high reactivity of the lonely electron for passivation with different species. Another, more versatile option, is to substitute the $\mathrm{Si}_{l}$ atom with another element during the epitaxial growth. Indeed, there are already examples where the electronic structure of epitaxial graphene can be tuned by the introduction of foreign species during the growth process [20-22].

Let us first discuss briefly the passivation option. Given the strong $\mathrm{Si}_{b}-\mathrm{C}_{b}$ bonding we have considered passivation only by small atomic species: $\mathrm{H}$ and Li. Larger atoms would disrupt the buffer layer geometry since they would not be compatible with the relative small volume around the $\mathrm{Si}_{l}$ atom. The main effect of the $\mathrm{H}$ adsorption is the disappearance of the interface state, as shown in Fig. 2(b). As one would expect, in the H-passivated system there is no charge transfer, the Dirac point of the first graphene monolayer lies exactly at the Fermi level and the geometry of the buffer layer is hardly affected. In the Li-passivated system on the contrary, the Li atom lies much closer to the $\mathrm{C}$ atoms in the buffer layer, and although the overall bonding geometry is not affected, the electronic structure shows some peculiar differences. In particular, one can note in Fig. 2(c) that different interface states appear to dominate the band structure. In fact, three additional bands appear (two in conduction and one in valence) with a tripledegenerate point in $\Gamma$ and a quasilinear dispersion in the vicinity of the Fermi level. These states arise from the hexagonal arrangement of the $\mathrm{Li}$ atoms on the 2D surface unit cell: indeed, Li atoms sit in a hcp supersymmetry and due to their relatively larger size the mutual interaction across the neighboring cells gives rise to the additional electronic bands [23]. Indeed, they form an extra electronic layer superimposed to the graphene on top, and provide an additional degree of freedom to tune the properties of the buffer. Above the buffer layer, the first graphene monolayer is characterized by a charge transfer of $1.31 \times 10^{13} \mathrm{~cm}^{-2}$ that pushes the Dirac point $0.47 \mathrm{eV}$ below the Fermi level making the system $n$-type.

The effect of passivation is not limited to a control of charge and orbitals at the interface, but affects also the macroscopic parameters central to band engineering. In fact, it is evident that different passivating species (or no passivation at all) can change the alignment between the energetic position of the Dirac point and the valence band edge on the $\mathrm{SiC}$ substrate (the valence band offset). In practice, by controlling the interfacial dipole by modifying the geometry of the buffer layer, one can gain control of the band alignment at the heterojunction. This effect is even more evident when we consider the option of substituting the $\mathrm{Si}_{l}$ atom with different surface impurities. We have considered the modification of the buffer layer by the substitution of the Si lonely atom with some of the most common SiC dopants: Al, B, P, and N.

In Fig. 3, we summarize the electronic band structure of the system. There are no partially occupied interface states, so that the Fermi level is not pinned, but in the case of group $\mathrm{V}$ impurities, $\mathrm{P}$ and $\mathrm{N}$, there is a filled interface state 
below the Fermi energy. The band structure of these 4 systems shows a variation of the valence band offset upon surface chemistry. Valence band offset, $\Delta E_{V}$, the energetic alignment between the valence band edge of $\mathrm{SiC}$ and the Fermi level, is a fundamental measure of the properties of the carrier transport at solid-state interfaces. We go from B doping with a valence band offset of $1.32 \mathrm{eV}$ to the $0.06 \mathrm{eV}$ of the system with $\mathrm{Al}$. The system with $\mathrm{N}$ has band offset of $1.30 \mathrm{eV}$ whereas the system with $\mathrm{P}$ has a band offset $1.44 \mathrm{eV}$. This variation clearly demonstrates that one can obtain the control and manipulation of the electrostatic potential profile via the tuning of the surface dipoles of the interface phase [5]. The Löwdin charges [24], a measure of the amount of charge localized around a given atomic site, can give us a quantitative estimate of the variation in the surface charges as they correlate with the interface dipoles. We calculated the Löwdin charges on each atom of the system with the Löwdin scheme used in the QUANTUM-ESPRESSO package. In particular, we observe a clear difference in the $\mathrm{B}$ and $\mathrm{Al}$ case (both group III atoms with a nominal +3 charge): indeed $\mathrm{B}$ shows a charge of 2.77 whereas the value is substantially lower, 2.03 , in the $\mathrm{Al}$ case. This variation is further enhanced by a countervariation of the charge of the $\mathrm{C}$ atoms in the subsurface layer: 4.82 in the B-doped system and 5.1 in the $\mathrm{Al}$ case. This variation is clearly correlated with the variation of the interface dipoles induced by the change in bonding char-
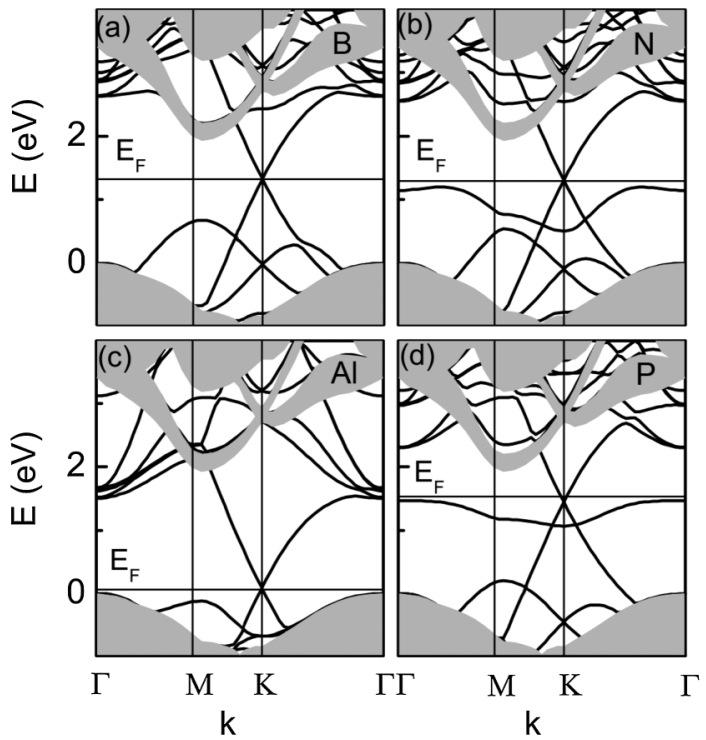

FIG. 3. The electronic band structures of the monolayer graphene and buffer layer on top of $\mathrm{SiC}$ substrate with $\mathrm{Si}_{l}$ atom is replaced by (a) B, (b) N, (c) Al, and (d) P. The valence band offsets $\quad(\mathrm{B} \rightarrow 1.32 \mathrm{eV}, \quad \mathrm{N} \rightarrow 1.30 \mathrm{eV}, \quad \mathrm{Al} \rightarrow 0.06 \mathrm{eV}, \quad \mathrm{P} \rightarrow$ $1.44 \mathrm{eV}$ ) show a clear variation upon surface chemistry. The surface chemistry also controls the charge transfer between the graphene layer and the substrate, as it is described in the text. The shaded area is the surface projected bulk band structure of $\mathrm{SiC}$ and all the energies are measured relative to the maximum of the valence band in bulk $\mathrm{SiC}$. acteristics and nominal ionic charge on the different atomic sites. Indeed the variation between the $\mathrm{Al}$ and $\mathrm{B}$ case is explained by the relative difference in electronegativity of the species, and a similar reasoning applies for $\mathrm{N}$ and $\mathrm{P}$, although now the induced dipoles and charge transfers occur in opposite direction. It is important to note that the high band offset is observed in the intrinsic interfaces with no passivation of the lonely atom, when the dangling bond electron dominates the interface dipole. These results have been obtained assuming a complete substitution of all the $\mathrm{Si}_{l}$ atoms in the $R 3$ cell (corresponding to a nominal coverage of $1 / 3$ of a monolayer). Clearly, by dosing the appropriate amount of dopants one could obtain a continuous variation of the band offset in a range as large as $1.5 \mathrm{eV}$. Careful observation of the electronic band structures in Fig. 3 suggests that by varying the surface chemistry, one can change the amount of charge transfer between the graphene layer and the substrate. Our preliminary calculations show a change in the direction of charge transfer with the graphene layer doping going from $n$ type to $p$ type [25]. Except for the P-substituted system in which the Dirac point lays $0.08 \mathrm{eV}$ below the Fermi energy, the calculated charge transfer does not shift the Dirac point significantly. While the amount of charge transfer for the $p$-type system is too small to make a significant impact in the system, our results suggest a possible pathway for controlling the electron and hole doping in the epitaxial graphene, which, however, requires further and more detailed studies [26].

Finally, we have investigated whether one can induce magnetization on the graphene layer via suitable manipulation of the buffer layer. Magnetism in graphene is currently the subject of intense investigation efforts, both theoretically and experimentally [27-32]. And, indeed, itinerant magnetism has been predicted to occur in the carbon buffer layer upon particular geometrical configuration of the interface phase [31]. Here we expand upon this idea and demonstrate that not only one can enforce particular electrostatic boundary conditions via the tuning of the buffer layer, but that the versatility of this approach is not limited to only charge, but can be extended to spin in a straightforward manner. We have chosen to incorporate one $\mathrm{Mn}$ atom in two different configurations in our interface geometry: first, we just replaced the $\mathrm{Si}_{l}$ atom as we have done previously with the group III and V dopants. In this geometry, the $\mathrm{Si}_{b}-\mathrm{C}_{b}$ distance remains at $2.0 \AA$, and the $\mathrm{C}_{b}-\mathrm{C}_{g}$ distance gets larger (between 4.05 and $4.31 \AA$ ) [26]. However, the electronic band structure for both majority and minority spin carriers in the system is not affected by the spin polarization of the buffer layers, and maintains its linear dispersion at the Dirac point. The electronic band structure reveals the appearance of one majority spin surface state close to the Fermi level while no minority spin surface states are found in the same energy region, while the graphene layer remains unpolarized. However, it would be certainly desirable to be able to induce a spin polariza- 

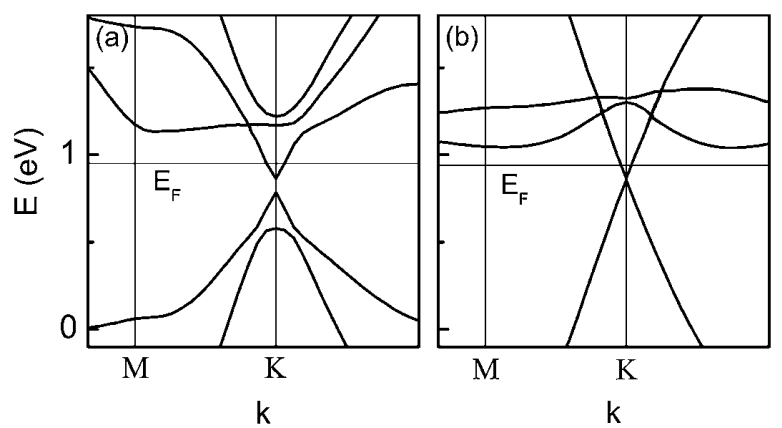

FIG. 4. Spin-polarized electronic band structures of the (a) majority and (b) minority spins of the monolayer graphene and buffer layer on top of $\mathrm{SiC}$ substrate with one of the $\mathrm{C}$ atoms in the $\mathrm{C}_{b}$ layer which is covalently bonded to $\mathrm{Si}_{b}$ atom, is replaced by a Mn atom. The system with Mn impurities in the buffer layer shows half-metallic behavior with a band gap opening of $\sim 80 \mathrm{meV}$ for majority spins at the Dirac point.

tion and itinerant magnetism on the graphene rather than on the buffer layer. To this aim we designed a geometry where the $\mathrm{Mn}$ atoms replaces one of the $\mathrm{C}_{b}$ atoms in the buffer layer. The $\mathrm{Mn}$ atom is covalently bonded to the $\mathrm{Si}$ atom of the substrate with a bond length of $2.66 \AA$. Because of larger atomic radius of $\mathrm{Mn}$, the buffer layer relaxes outwards, leaving two unbounded $\mathrm{Si}$ atoms in the substrate that we farther saturated with two $\mathrm{H}$ atoms.

In Fig. 4 we show the band structure for the majority (4a) and minority (4b) spin electrons. It is clear from these data that the modification induced by the $\mathrm{C}$ substitution with $\mathrm{Mn}$ in the buffer layer could induce a half-metallic behavior in the graphene layer above under specific gating of the sample [33]. Indeed, the most remarkable property in this band structure is the opening, in the graphene bands, of an energy gap $(\sim 80 \mathrm{meV})$ for the majority spins, while minority spins still maintain the linear dispersion typical of the Dirac point.

This work was supported, in part, by the DARPA/HRL CERA, SRC/FCRP FENA, and U.S. ARO. M. B. N. wishes to acknowledge partial support from the Office of BES, U.S. DOE at ORNL under Contract No. DE-AC0500OR22725 with UT-Battelle, LLC. Calculations have been carried out at NCCS-ORNL.

*Corresponding author. mbnardelli@ncsu.edu

[1] W. A. de Heer et al., Solid State Commun. 143, 92 (2007).

[2] F. Varchon et al. Phys. Rev. Lett. 99, 126805 (2007).

[3] H. Hibino et al., Phys. Rev. B 80, 085406 (2009).

[4] J. Hass et al., J. Phys. Condens. Matter 20, 323202 (2008).

[5] S.-M. Choi and S.-H. Jhi, Appl. Phys. Lett. 94, 153108 (2009).

[6] P. Xiangyang and A. Rajeev, Nano Lett. 8, 4454 (2008).

[7] S. Kim, J. Ihm, H. J. Choi, and Y. -W. Son, Phys. Rev. Lett. 100, 176802 (2008).
[8] M. Hupalo, E. H. Conrad, and M. C. Tringides, Phys. Rev. B 80, 041401(R) (2009).

[9] J. Penuelas et al., Phys. Rev. B 79, 033408 (2009).

[10] P. Shamella and S. K. Nayak, Appl. Phys. Lett. 94, 032101 (2009).

[11] R. A. McKee et al., Science 300, 1726 (2003).

[12] M. Nuñéz and M. Buongiorno Nardelli, Phys. Rev. B 73, 235422 (2006).

[13] I. Forbeaux, J.-M. Themlin, and J.-M. Debever, Phys. Rev. B 58, 16396 (1998).

[14] C. Berger et al., Science 312, 1191 (2006).

[15] J. Hass et al., Appl. Phys. Lett. 89, 143106 (2006).

[16] T. Ohta et al., Science 313, 951 (2006).

[17] P. Mallet et al., Phys. Rev. B 76, 041403(R) (2007).

[18] A. Mattausch and O. Pankratov, Phys. Rev. Lett. 99, 076802 (2007).

[19] QUANTUM-ESPRESSO is a community project for highquality quantum-simulation software, based on densityfunctional theory, and coordinated by Paolo Giannozzi. See www.quantum-espresso.org. Technical specification for the calculations are as follows: $12 \times 12 \times 1$ Monkhorst-Pack grid for Brillouin zone integration; 35 Ry energy cutoff for the plane waves basis expansion; ultrasoft pseudopotentials; Perdew-Burke-Ernzerhof (PBE) generalized gradient approximation for the exchange correlation; a vacuum region of $18 \AA$ separates the slabs in the supercell geometry. Geometries have been optimized to forces less than $0.025 \mathrm{eV} / \AA$ and a dipole correction is used to cancel the artificial dipole field due to the polarization of different surface terminations [20].

[20] C. Riedl et al., Phys. Rev. Lett. 103, 246804 (2009).

[21] I. Gierz et al., Nano Lett. 8, 4603 (2008).

[22] N. P. Guisinger et al., Nano Lett. 9, 1462 (2009).

[23] N. W. Aschroft and N.D. Mermin, Solid State Physics (Saunders, Philadelphia, 1976).

[24] D. Sanchez-Portal, E. Artacho, and J. M. Soler, Solid State Commun. 95, 685 (1995).

[25] The amount of charge transferred to and from the graphene layer for the four systems is the following: $\mathrm{B},-0.598 \times 10^{12} \mathrm{~cm}^{-2} ; \mathrm{N},-1.98 \times 10^{12} \mathrm{~cm}^{-2}$; $\mathrm{Al},-3.03 \times 10^{12} \mathrm{~cm}^{-2}$; and $\mathrm{P}, 6.29 \times 10^{12} \mathrm{~cm}^{-2}$, respectively. Note the change in the direction of the charge transfer depending on the surface chemistry.

[26] It should be noted that the amount of charge transfer could be affected by the choice of exchange and correlation functional in DFT and the neglect of dispersion forces.

[27] M. Nishioka and A. M. Goldman, Appl. Phys. Lett. 90, 252505 (2007).

[28] T. Nikolaos et al., Nature (London) 448, 571 (2007).

[29] Y. Mao, J. Yuan, and J. Zhong, J. Phys. Condens. Matter 20, 115209 (2008).

[30] H. Sevincli et al., Phys. Rev. B 77, 195434 (2008).

[31] A. Ramasubramaniam, N. V. Medhekar, and V. B. Shenoy, Nanotechnology 20, 275705 (2009).

[32] K. T. Chan, J. B. Neaton, and M. L. Cohen, Phys. Rev. B 77, 235430 (2008).

[33] The Mn substitution induces a charge transfer on the graphene layer that shifts the Fermi energy above the Dirac point. However, the relative position of the Fermi level can be controlled via external gating and the peculiar half-metallic character that ensues can be effectively exploited. 